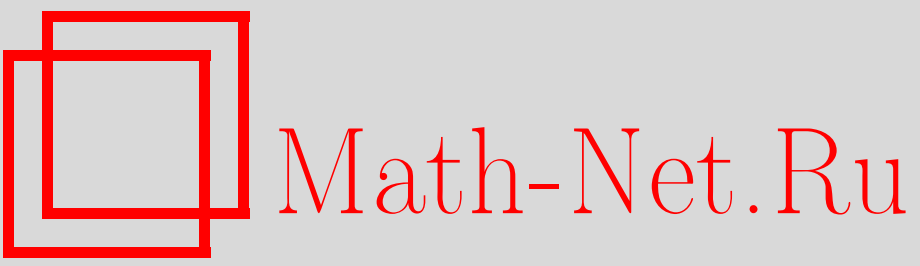

В. В. Вьюгин, Эргодическая теорема для индивидуальной случайной последовательности, УМН, 1996, том 51, выпуск 6, 191-192

DOI: https://doi.org/10.4213/rm1022

Использование Общероссийского математического портала Math-Net.Ru подразумевает, что вы прочитали и согласны с пользовательским соглашением

http://www . mathnet.ru/rus/agreement

Параметры загрузки:

IP : 44.207 .124 .84

26 апреля 2023 г., 06:42:01 


\title{
ЭРГОДИЧЕСКАЯ ТЕОРЕМА ДЛЯ ИНДИВИДУАЛЬНОЙ СЛУЧАЙНОЙ ПОСЛЕДОВАТЕЛЬНОСТИ
}

\author{
В.В. Вьюгин
}

Пусть последовательность случайных величин $\xi_{1}, \xi_{2}, \ldots, \xi_{n}, \ldots$ является стационарной (в узком смысле), т.е. для любого $k \geqslant 1$ и измеримого $B$

$$
\mathrm{P}\left\{\left(\xi_{1}, \xi_{2}, \ldots\right) \in B\right\}=\mathrm{P}\left\{\left(\xi_{k}, \xi_{k+1}, \ldots\right) \in B\right\} .
$$

Эргодическая теорема утверждает, что если $\mathrm{E}\left|\xi_{1}\right|<\infty$, то предел

$$
\lim _{n} \frac{\xi_{1}(\omega)+\xi_{2}(\omega)+\cdots+\xi_{n}(\omega)}{n}
$$

существует почти всюду [4]. При этом классическая теория вероятностей не позволяет точно описать класс тех $\omega$, для которых предел (1) существует. Положение усугубляется также тем, что эргодическая теорема, в определенном смысле, оказывается "неконструктивной". Мы будем использовать понятие алгоритмически вычислимой функции [3]. Последовательность случайных величин $\eta_{1}, \eta_{2}, \ldots$ сходится по вероятности к случайной величине $\eta$ алгоритмически эффективно, если существует целочисленная алгоритмически вычислимая функция $m(\delta, \varepsilon)$ с рациональными аргументами такая, что для всех рациональных $\delta>0$ и $\varepsilon>0$ будет выполняться $\mathrm{P}\left\{\left|\eta_{n}-\eta\right|>\delta\right\}<\varepsilon$ для всех $n \geqslant m(\delta, \varepsilon)$.

В качестве вероятностного пространства будем рассматривать $(\Omega, \mathscr{F}, \mathrm{P})$. Здесь $\Omega=I^{\infty}$, где $I=\{0,1, \ldots, l\}, l \geqslant 1, \mathscr{F}$ - борелевское поле, порожденное шарами вида $\Gamma_{x}=\left\{\omega: \omega_{1}=x_{1}\right.$, $\left.\ldots, \omega_{k}=x_{k}\right\}$, где $k$ - натуральное число, $x=x_{1} \ldots x_{k}, x_{1} \in I, \ldots, x_{k} \in I, \omega_{k}$ обозначает $k$-й член последовательности $\omega$. Мера Р назьвается вычислимой, если существует алгоритм, вычисляющий по $x$ и рациональному $\varepsilon>0$ рациональное приближение величины $\mathrm{P}\left(\Gamma_{x}\right)$ с точностью до $\varepsilon$.

Теорема 1. Существует вычислимая стационарная мера, для которой сходимость по вероятности $\frac{1}{n} \sum_{k=1}^{n} \omega_{k} \rightarrow f(\omega)$ не является алгоритмически әффективной (здесь $f(\omega)$ - предельная функчия, существующая по әргодической теореме).

Поскольку сходимость почти всюду эквивалентна сходимости

$$
\mathrm{P}\left\{\omega: \sup _{m \geqslant n}\left|\frac{1}{m} \sum_{k=1}^{m} \omega_{k}-f(\omega)\right|>\delta\right\} \rightarrow 0
$$

для любого $\delta>0$, сходимость почти всюду также не является алгоритмически эффективной.

Тем не менее, эргодическая теорема допускает алгоритмически удовлетворительную форму в рамках предложенного А. Н. Колмогоровым алгоритмического подхода к обоснованию теории вероятностей и теории информации [2]. Точное математическое понятие случайной последовательности позволяет формулировать законы теории вероятностей для индивидуальных последовательностей и находить новые "алгоритмические" формулировки и доказательства этих законов. Яркий пример такого рода - найденное Вовком [1] алгоритмическое доказательство закона повторного логарифма для случайных последовательностей.

Для определения случайной последовательности выделяется свойство "типичности", которое утверждает, что случайная последовательность должна принадлежать любому "разумному" большинству, которое определяется алгоритмически [3]. Пусть Р - некоторая вычислимая мера на $\Omega$. Множество $M \subseteq \Omega$ имеет Р-меру 0 , если для любого рационального $\varepsilon>0$ существует такая последовательность шаров $\Gamma_{x(1)}, \ldots, \Gamma_{x(n)}, \ldots$, что для $U_{\varepsilon}=\bigcup \Gamma_{x(n)}$ будем иметь $M \subseteq U_{\varepsilon}$ и $\mathrm{P}\left(U_{\varepsilon}\right)<\varepsilon$. Р-нулевое множество $M$ называется әффективно нулевым, если

Работа выполнена при частичной поддержке INTAS (грант № 93-0893). 
существует алгоритм, который по произвольному рациональному $\varepsilon>0$ перечисляет последовательность $x(1), \ldots, x(n), \ldots$, порождающую такое множество $U_{\varepsilon}$. Мартин-Лёф доказал, что для произвольной вычислимой меры $\mathrm{P}$ существует максимальное по включению эффективно Р-нулевое множество - его дополнение называется конструктивным носителем меры $\mathrm{P}$. Бесконечная последовательность называется типической относительно меры $\mathrm{P}$ (случайной в смысле Мартин-Лёфа), если она лежит в конструктивном носителе меры $\mathrm{P}$ [3].

Вопрос об истинности эргодической теоремы для типических последовательностей был поставлен в [5].

Теорема 2. Для любой вычислимой стационарной меры $\mathrm{P}$ существует интегрируемая функиия $f$ такая, что $\mathrm{E}(f)=\sum_{i=0}^{l} i \mathrm{P}\left\{\omega: \omega_{1}=i\right\}$ u

$$
\lim _{n} \frac{1}{n} \sum_{k=1}^{n} \omega_{k}=f(\omega)
$$

для любой типической относительно меры $\mathrm{P}$ последовательности $\omega$, кроме этого, $f(T \omega)=f(\omega)$, где $T-$ сдвиг $\left(\right.$ т.е. $(T \omega)_{i}=\omega_{i+1}$ для всех $\left.i\right)$. Если, к тому жсе, сдвиг $T$ әргодичен, то $f(\omega)=\mathrm{E}(f)$ ( $\mathrm{E}$ - символ математического ожидания).

\section{СПИСОК ЛИТЕРАТУРЫ}

[1] Вовк В. Г. // Теория вероятн. и ее примен. 1987. Т. 32. № 3. С. 456-468. [2] Колмогоров А. Н. // Проблемы передачи информации. 1969. Т. 5. №3. С. 3-7. [3] Колмогоров А. Н., Успенский В.А. // Теория вероятн. и ее примен. 1987. Т. 32. № 3. С. 425-455. [4] Ширяев А. Н. Вероятность. М.: Наука, 1980. [5] Lambalgen M. Random sequences. Amsterdam: Academish Proefschri't, 1987.

Институт проблем

передачи информации РАН
Принято редколлегией 06.09 .1996 